Article

\title{
Tesla Bladed Pump (Disc Bladed Pump) Preliminary Experimental Performance Analysis
}

\author{
Yaguang Heng ${ }^{1,2} \oplus$, Yuming Han ${ }^{1}$, Huiyu Zhang ${ }^{1}$, Weibin Zhang ${ }^{1}$, Gérard Bois ${ }^{1,3}$, \\ Qifeng Jiang ${ }^{1, *} \mathbb{D}$, Zhengwei Wang ${ }^{2, *}$ and Xiaobing Liu ${ }^{1}$
}

1 Key Laboratory of Fluid and Power Machinery, Xihua University, No. 9999 Hongguang Avenue, Pidu District, Chengdu 610039, China; hengyaguang@gmail.com (Y.H.); stuhanyuming@gmail.com (Y.H.); zhanghuiyu600@gmail.com (H.Z.); zhangwb@mail.xhu.edu.cn (W.Z.); gerard.bois@ensam.eu (G.B.); liuxb@mail.xhu.edu.cn (X.L.)

2 Department of Energy and Power Engineering, Tsinghua University, No. 30 Shuangqing Road, Haidian District, Beijing 100084, China

3 CNRS, ONERA, Arts et Metiers Institute of Technology, Centrale Lille, UMR 9014-LMFL-Laboratoire de Mécanique des Fluides de Lille-Kampé de Fériet, University of Lille, F-59000 Lille, France

* Correspondence: qifeng.jiang@mail.xhu.edu.cn (Q.J.); wzw@mail.tsinghua.edu.cn (Z.W.)

Received: 15 July 2020; Accepted: 12 September 2020; Published: 17 September 2020

check for updates

\begin{abstract}
A preliminary experimental work on a centrifugal pump model specifically designed to transport slurry and multiphase flows is presented. The impeller design corresponds to the so-called "Tesla Bladed Pump" adapted from an existing Discflow ${ }^{\mathrm{TM}}$ pump design for the petroleum deep-sea application. The overall performance results of such a specific pump design are presented for different rotational speeds and discussed in relation to affinity laws coefficients. The results show that the performance of the tested disc pump strongly differs from the conventional centrifugal bladed pump. A one-dimensional approach using complete Euler relation is used to explain the differences in the present case. Moreover, it has been found that available results in open literatures do not correspond to the real optimum conditions, more detailed research work must be performed to get a better understanding on this kind of bladed disc pump.
\end{abstract}

Keywords: centrifugal; tesla bladed pump; multiphase flow; experimental analysis; performance characteristics

\section{Introduction}

The initial basic idea concerning this specific pump device category is based on a simple first design invented around 1850 in the US (see Figure 1a). This first disc pump was built using a closed cylinder pierced by several holes to ensure flow rate. It operates solely on the boundary layer viscous drag principle. Around 1900, Nicola Tesla developed a new version that enables pumping of high-viscosity fluids using centrifugal effects between two small spacing corotating discs. This kind of device can generally pump a small amount of flow rate with small head coefficient compared with classical rotating centrifugal pumps. By adding several corotating discs (as shown in Figure 1b, so-called "Discpac" design, patented in 1988 [1]), one can only increase the flow rate, but there are still low head values. Based on the design considerations proposed by Cappelino et al. [2] and combined effects of viscous driven forces acting on tesla disc pumps, it is possible to design a specific tesla bladed pump impeller, using corotating discs equipped with small blade heights, which may reach higher flow rates and head values [3-5]. An example is given by a pump model tested by Yin [6], which is capable of reaching a total head of $120 \mathrm{~m}$ (of water) with a flow rate of $240 \mathrm{~m}^{3} / \mathrm{h}$ at $2900 \mathrm{rpm}$. These values can be obtained using an impeller outlet diameter of $0.32 \mathrm{~m}$ and purely radial straight blade shapes, 
as shown in Figure 1c. The maximum efficiency is generally lower than $50 \%$ due to the quite simple and crude impeller design characteristics.

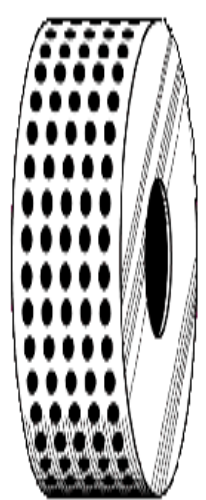

(a)

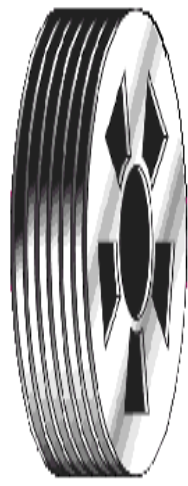

(b)

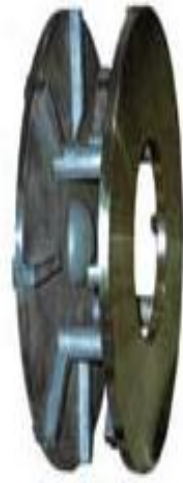

(c)

Figure 1. Evolution of disc pump impeller concepts. Flow enters from the right parallel to the rotational axis. (a) Rotating cylinder with holes. (b) Tesla disc pack (without radial blades). (c) Bladed disk pump (radial blades with large gap).

This arrangement can be considered equivalent to twin face to face open centrifugal pump impellers that rotate at the same angular speed with a large gap in between. Figure 2 shows the impeller arrangement used for the present study, the so-called tesla bladed disc (TBD) pump.

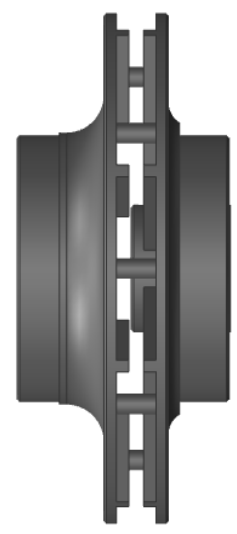

Figure 2. Present tesla bladed disk (TBD) impeller (the flow direction can be seen in Figure 3).

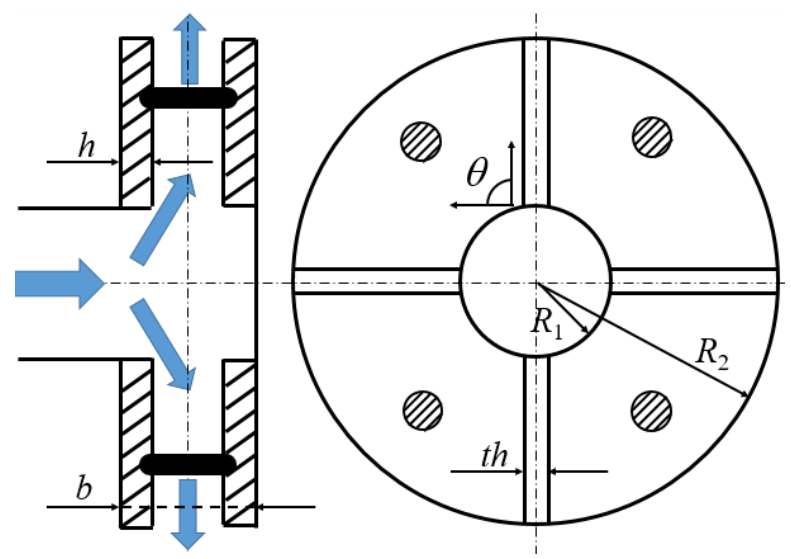

Figure 3. Sketch of a disc bladed impeller (four radial blades are represented in this figure instead of eight). 
Only few studies have been published on this kind of pump, mainly because of the lack of interest due to low performance and global efficiency values. The corresponding unusual and quite crude design, compared with classical impeller one, however allows the pumping and transport of a large variety of multiphase sludge materials. For petroleum applications, these pumps usually work at high inlet pressure to avoid cavitation problems. Most of the pump models come from industrial patents and related comparisons between experiments and computational fluid dynamics (CFD) approaches are quite rare and not always well documented.

Some interesting analyses have been however performed by researchers at the China University of Petroleum (East China) [6-10], looking at the effects of impeller geometrical parameters on the pump performance. Another paper from Je and Kim [11] explores the effects on the blade clearance gap, using only CFD results on another pump model case. The relevant literatures are listed in Table 1 and the parameters that have been explored are illustrated in Figure 3.

Table 1. Literatures related to the Tesla bladed pump.

\begin{tabular}{cccccc}
\hline Reference & $\boldsymbol{R}_{\mathbf{2}} / \boldsymbol{R}_{\mathbf{1}}$ & $\mathbf{2 h / b}$ & $\boldsymbol{N}$ & $\boldsymbol{\theta}$ & $\boldsymbol{t h}$ \\
\hline Yin [6] & & & & $\sqrt{ }$ & \\
Gao [7] & $\sqrt{ }$ & $\sqrt{ }$ & $\sqrt{ }$ & & \\
Zhou [8] & $\sqrt{ }$ & $\sqrt{ }$ & $\sqrt{ }$ & & $\sqrt{ }$ \\
Chen [9] & & & $\sqrt{ }$ & & $\sqrt{ }$ \\
Zhang [10] & $\sqrt{ }$ & $\sqrt{ }$ & & & \\
Je and Kim [11] & & $\sqrt{ }$ & & & \\
\hline
\end{tabular}

Since the Tesla bladed pump utilizes pressure and viscous forces to transport the fluids towards the impeller outlet section, classical conclusions coming from usual centrifugal pump design can be partially applied to the disc pump. For example, the results presented by Gao [7], Zhou [8] and Zhang [10] achieve consistent conclusions that the disc impeller with larger values of radius ratio $R_{2} / R_{1}$ and rotational speed $N$ provides higher lift head, which is a typical conclusion for centrifugal pumps. However, some inconsistent conclusions also can be found in these studies. The results presented by Gao [7] indicated that the disc pump lift head reaches its maximum value when $2 h / b$ $=0.3$, whereas Zhou [8] and Zhang [10] state that the pump lift head still increases linearly with the increase of $2 h / b$; the extracted data are shown in Figure 4. Besides, Yin [6] suggests a blade angle $\theta$ (see Figure 3) between $25^{\circ}$ to $35^{\circ}$ to reach better pump performance; however, the reason is unclear. Most of these studies only focused on the external pump performance behaviors for engineering applications, but rarely reveal the underlying flow characteristics of this kind of disc bladed pump. Analytical one-dimensional approaches using semiempirical criteria on slip factor and loss coefficient have not been already performed.

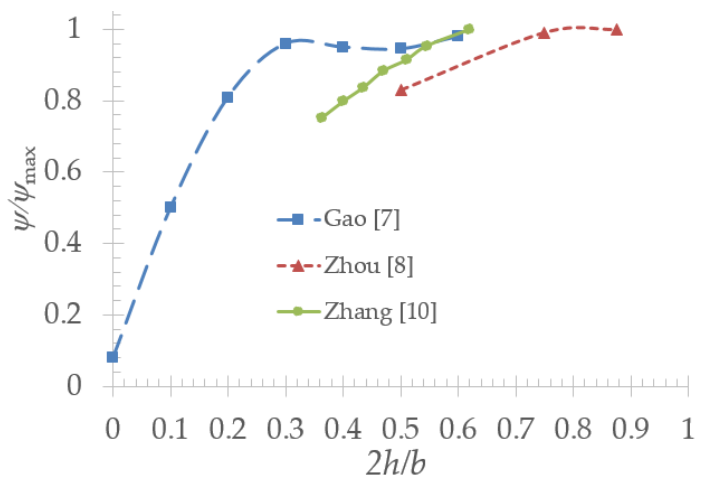

Figure 4. Tesla bladed pump lift head ratio versus coefficient $h / b$. (extracted from Gao [7], Zhou [8] and Zhang [10]). 
For the present study, a tesla bladed pump impeller is placed inside a conventional existing centrifugal pump hub-shroud-volute casing. Overall performance nondimensional coefficients are experimentally obtained and analyzed for several rotational speeds using water as the working fluid. The aim of this work is to understand part of the mean flow properties through specific data reduction assumptions derived for present unusual impeller geometry and define both experimental and numerical research strategies to get better understanding of local flow features. The final objective of this work concerns the capability of such a design to handle inlet two-phase flow pumping applications with extended pump shut-off performance compared with existing designs.

\section{The Impeller Pump Model Main Parameters}

The present TBD geometrical parameters are given here:

Inlet tip radius
Outlet radius
Number of blades
Inter blade clearance gap
Blade height
Blade angle
Impeller channel width

$$
\begin{aligned}
& R_{1}=50 \mathrm{~mm} \\
& R_{2}=100 \mathrm{~mm} \\
& Z=8 \\
& c l=8 \mathrm{~mm} \\
& h=4 \mathrm{~mm} \\
& \theta=90^{\circ}, \text { radial blade } \\
& b=16 \mathrm{~mm}
\end{aligned}
$$

The relative distance between the two discs $b_{2} / R_{2}$ is lower compared with other ones previously studied by Yin [6] and Je and Kim [11]. For convenience, the present chosen TBD impeller dimensions have been adapted to be directly inserted in an existing impeller and volute casing. Consequently, the front and rear casing chambers' designs are imposed and not necessary well adapted to the present impeller geometry. This means that strong flow leakage may exist with bad volumetric efficiency due to the eight axial thrust equilibrium holes that have been drilled on the hub impeller side wall.

Blade shape profiles are very simple ones corresponding to eight equally spaced radial straight blades that are fixed on each disc. This design allows to ensure no blockage effects at the impeller inlet section in case of inlet slurry condition, but this probably leads to high local losses due to large values of the angle of attack and blade thickness blockage whatever the flow rate is.

In addition, the volute size has been kept the same and corresponds to a classical backswept impeller design. Consequently, it is expected that the volute is not well adapted for the present TBD impeller design because of an expected quite small impeller outlet absolute angle value. The prototype used in experiments is shown in Figure 5.
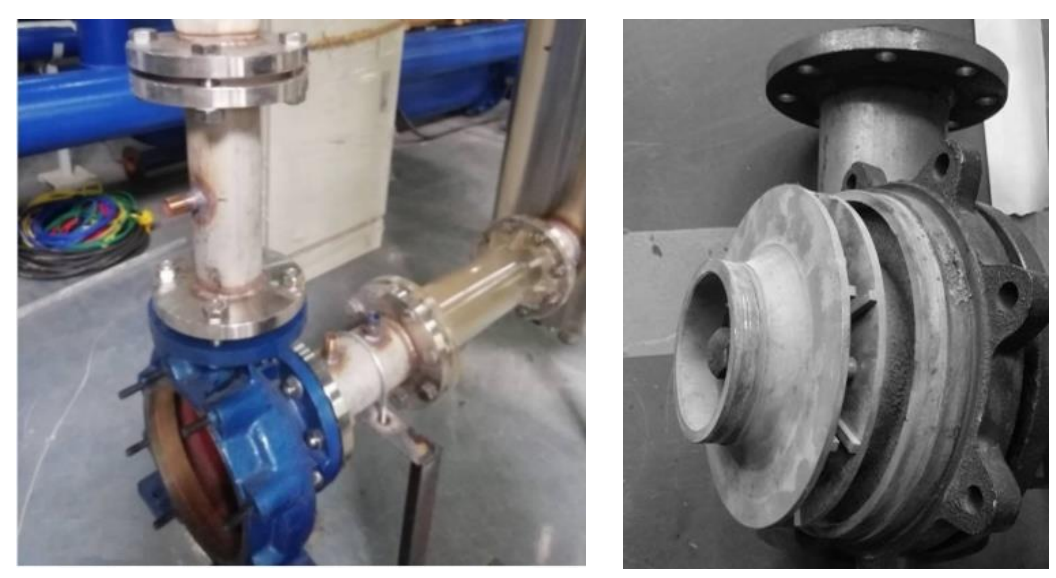

Figure 5. TBD pump casing (left) and impeller (right). 


\section{Experimental Study}

\subsection{Experimental Set Up}

Figure 6 shows the test loop diagram with instrumentation. A tank (open to atmospheric pressure) with a volume of $3 \mathrm{~m}^{3}$ is placed to provide the recyclable fluid, valves are used to control the flow rate and two flow meters are placed upstream and downstream of the pump for flow rate evaluation. The use of the second flow meter concerns future experimental works linked to inlet two-phase flow conditions. Wall static pressure taps are placed in the front and outlet sections of the pump. Pump head is obtained by calculating the pressure difference between the wall static taps and adding the dynamic pressure assuming constant mean axial flow conditions at inlet and outlet pipe sections. Because of the high incidence angle, even for pump design conditions, TBD should be very sensitive to cavitation. Inlet pressure value is controlled to check possible inlet cavitation occurrence (see comments in Section 3.2). However, this topic is not included for the present analysis because the present test loop inlet tank is open to atmospheric conditions and does not allow inlet pressure control for cavitation tests.

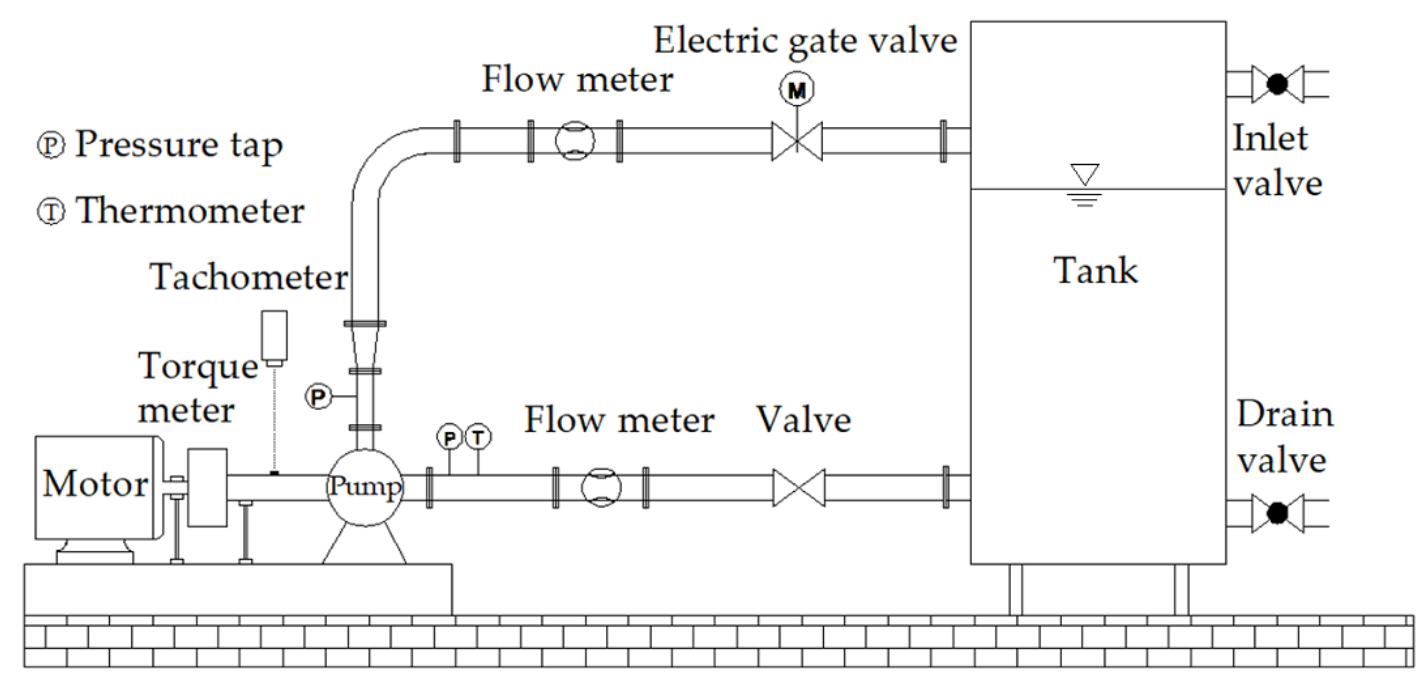

Figure 6. Test loop diagram: tank is open to atmospheric conditions, inlet valve is used to fill up the tank with water, and water level is measured and generally set at $2 \mathrm{~m}$.

Efficiency is obtained using a torque-meter device placed between the electric motor and the pump shaft. The rotational speed is also controlled by the same device. Measurement accuracies should be improved since flow rate and head values are presently obtained within a $5 \%( \pm 2.5 \%)$ error band due to strong unsteady pressure observed mainly at the pump inlet section. They are, however, considered to be acceptable to perform the preliminary analysis which is presented in the next sections.

Instruments uncertainty are the following:

- Volume flow rate transducer precision: $\pm 0.5 \%$; range: $0-100 \mathrm{~m}^{3} / \mathrm{h}$;

- Volume flow rate measuring error due to uncertainty and fluctuation: $\pm 2.5 \%$;

- Rotational speed transducer precision: $\pm 0.1 \%$; range: 0-3000 rpm;

- Rotational speed error (speed regulation): $\pm 0.5 \%$;

- Torque transducer precision: $\pm 0.2 \%$; Range: $0-1000 \mathrm{~N} \cdot \mathrm{m}$;

- Torque measuring error due to uncertainty and fluctuation: $\pm 1 \%$;

- Pump inlet and outlet pressure measuring device precision: $\pm 0.1 \mathrm{~m}$; range: 0-50 m;

- Pump head pressure precision: $\pm 0.2 \mathrm{~m}$, assuming pure axial velocity inside the inlet and outlet tube section in which static pressures are measured, and the corresponding error is $\pm 0.4 \mathrm{~m}$ for full scale. 
Each transducer precision comes from manufacturer indications. Pressure is calibrated using hydrostatic values at pump rest up to $3 \mathrm{~m}$, corresponding to $6 \%$ of the device's full range.

\subsection{Head Coefficient and Efficiency Curves Versus Flow Coefficient}

Figures 7 and 8 respectively show the evolutions of the head coefficient and the efficiency versus flow coefficient for four different rotational speeds of 1000, 1500, 2000 and $2900 \mathrm{rpm}$. The definition of each coefficient is given as follows:

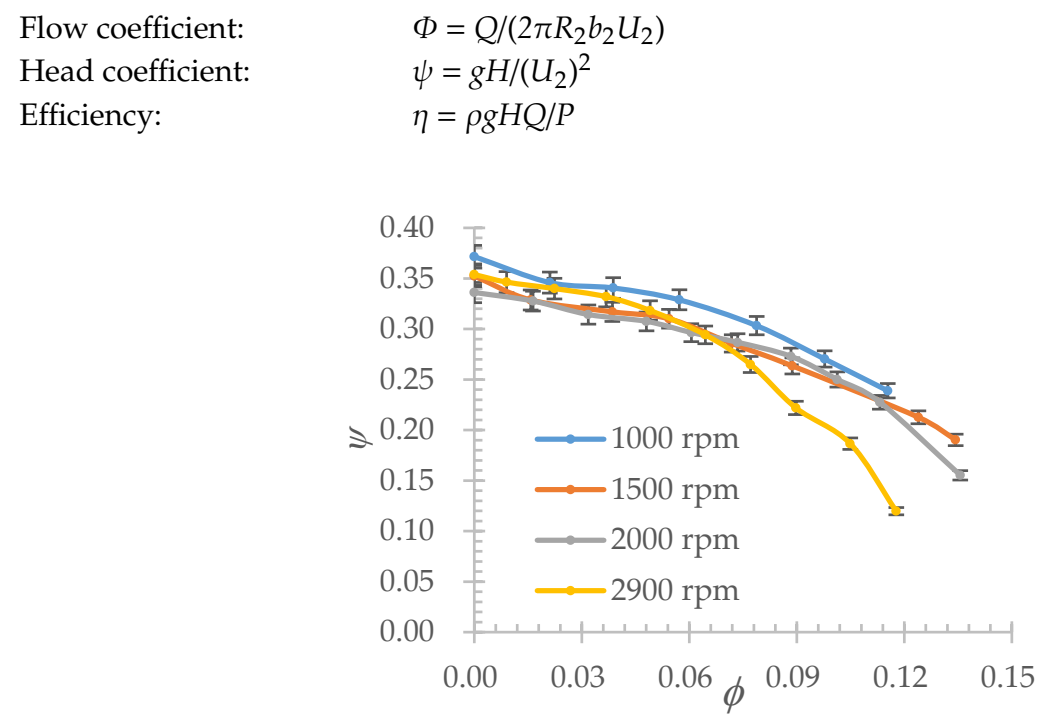

Figure 7. Head coefficient versus flow coefficient for several rotational speeds.

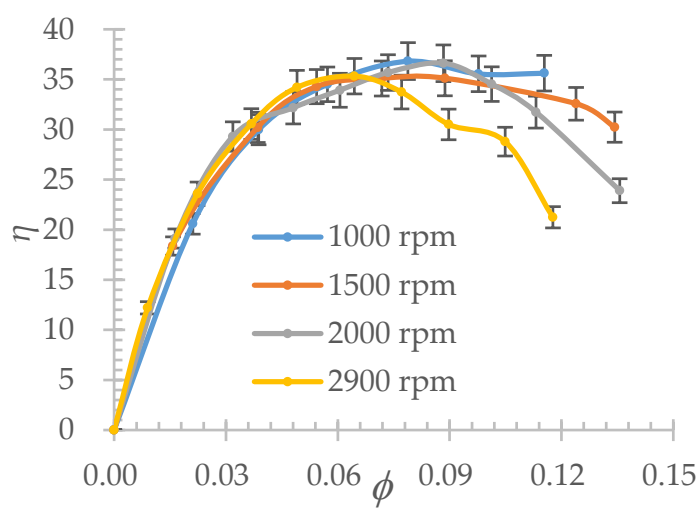

Figure 8. Global efficiency versus flow coefficient for several rotational speeds.

For a flow rate of $100 \mathrm{~m}^{3} / \mathrm{h}$ and a rotational speed of $2900 \mathrm{rpm}$, the corresponding uncertainty range for flow coefficient is: $\Phi=0.088 \pm 0.005$. For the head coefficient $\psi=0.30 \pm 0.08$ and for the efficiency, $\eta=0.35 \pm 0.03$.

Part of the head coefficients and efficiencies values do not have the same values and so, do not follow the usual affinity laws for conventional impeller designs. Head coefficients (Figure 7) are found to have higher values for decreasing rotational speeds. For large flow coefficients and rotational speed of $2900 \mathrm{rpm}$, head coefficient values rapidly decrease. In Figure 8, global efficiency values are always better after reaching the maximum point when rotational speed diminishes. The corresponding theoretical head shown in Figure 9, which combines both previous results, indicates that the input power per unit volume rate taken by the shaft is comparatively higher for $1000 \mathrm{rpm}$ compared with $2900 \mathrm{rpm}$. 


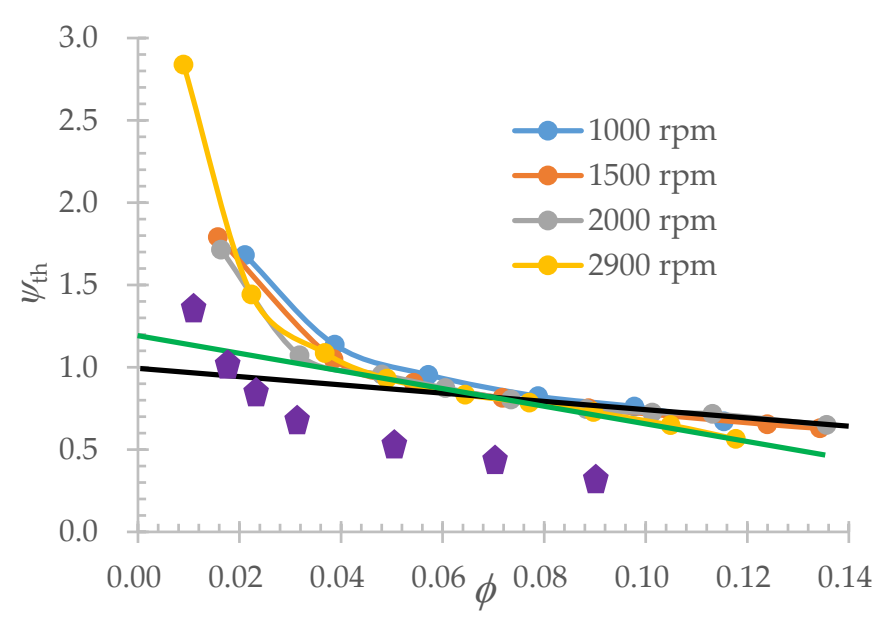

Figure 9. Theoretical head coefficient versus flow coefficient for four different rotational speeds (purple diamond symbols are extracted from the results published by Yin [6] for $2900 \mathrm{rpm}$ ).

Compared to the conventional centrifugal pump, the differences that can be seen in Figures 7 and 8 can be explained due to following reasons:

(a) The tested impeller design strongly differs from conventional design blade shape and blade arrangement including a large gap between each parallel disc. Thus, both pressure and viscous forces should have comparable relative effects compared with conventional designs for which pressure force work is generally much higher than viscous one.

(b) Consequently, for the lowest rotational speed (1000 rpm), part of the work given to the fluid may be governed by Reynolds number effects specifically along the flat disc region that rotates inside the casing chambers. These parts act like the original tesla disc pump. It is well-known that these kinds of pumps give more pressure at low rotational speeds due to laminar flow regime but need higher relative shaft power.

(c) For turbulent flow regimes, like the one that should be obtained at high rotational speeds, efficiency can more strongly depend on clearance flow mixing due to the increase of pressure difference between driving and driven impeller faces.

(d) For $2900 \mathrm{rpm}$, cavitation noise has been detected for high flow rates. The last three experimental points maybe affected by this problem because of measured low inlet static pressure values and should be ignored.

(e) Another effect cannot be neglected. It is related to leakage flows that occur inside both side chambers which are responsible to the so-called volumetric efficiency, this corresponds to the fact that the real flow rate that is driven by the impeller is bigger than the exhaust flow rate measured by the flow meter devices placed on the loop.

(f) Maximum efficiency point is slightly shifted towards higher flow coefficients for decreasing rotational speeds. It reaches a maximum value of 0.37 , for a flow coefficient value closed to 0.06 at $1000 \mathrm{rpm}$. Maximum efficiency values are relatively low, they are however quite comparable with the values obtained by Je and Kim [11] for the same relative blade height ratio $h / b$ (See Table 2) at design conditions. Also note that such performance curves can also be found for conventional designs of open impeller pump with a relative blade height value $h_{2} / b_{2}=0.9$, which is even bigger than the present case (Choi et al. [12], see Table 2).

(g) Flow coefficient values $\Phi$ given in [6] and [11] are quite low compared with the present case. This can be explained by the fact that efficiency curves are often flat ones and that maximum efficiency value have not been reached by these authors because of too much test loop losses that limit high flow rate experimental conditions. 
Table 2. List of several main pump characteristics used in literatures and the present study.

\begin{tabular}{cccccccccccc}
\hline Reference & $\boldsymbol{R}_{\mathbf{2}}$ & $\boldsymbol{R}_{\mathbf{2}} / \boldsymbol{R}_{\mathbf{1}}$ & $\boldsymbol{N}$ & $\boldsymbol{b}_{\mathbf{2}} / \boldsymbol{R}_{\mathbf{2}}$ & $\boldsymbol{h}_{\mathbf{2}} / \boldsymbol{b}_{\mathbf{2}}$ & $\boldsymbol{Z}$ & $\boldsymbol{\theta}_{\mathbf{2}}$ & $\boldsymbol{\theta}_{\mathbf{1}}$ & $\boldsymbol{\Phi}$ & $\boldsymbol{\Psi}$ & $\boldsymbol{\Omega} \mathbf{s}$ \\
\hline Yin [6] & 0.16 & 2.7 & 2900 & 0.33 & 0.28 & 8 & 90 & 90 & 0.026 & 0.48 & 0.34 \\
Je and Kim [11] & $0.11^{*}$ & 2.7 & 1750 & 0.33 & $0.25^{*}$ & $8^{*}$ & $90^{*}$ & $90^{*}$ & 0.04 & 0.38 & 0.316 \\
Present study & $0.10^{*}$ & 2.0 & $1000 / 2900$ & 0.16 & $0.25^{*}$ & $8^{*}$ & $90^{*}$ & $90^{*}$ & 0.08 & 0.28 & 0.74 \\
Choi et al. [12] & 0.06 & 4.0 & 700 & 0.15 & 0.888 & 6 & 90 & 90 & 0.04 & 0.4 & 0.20 \\
\hline
\end{tabular}

* These numbers correspond to common geometrical design parameters related to the present paper.

\subsection{Theoretical Head Coefficient Versus Flow Coefficient}

In Figure 9, the values defined by $\psi_{\text {th }}=\psi / \eta$ are plotted versus flow coefficient. Although results are not corrected by disc friction and leakage effects, especially when flow coefficient values are below 0.02 , one can consider that all results practically exhibit the same evolution for all four rotational speeds. Some experimental results on theoretical head values, extracted from Yin [6], have been added in purple diamond symbols on Figure 9. The curve slope is the same compared with the present experimental results, owing to uncertainty evaluation coming from the referred paper results.

\section{Discussions}

\subsection{Euler Head Coefficient Curve Development}

According to the ideal conservation law of angular momentum in rotating centrifugal pump, the Euler head $\left(H_{E}\right)$ or theoretical head can be expressed as:

$$
H_{E}=H_{\text {th }}=\frac{\vec{V}_{2} \times \vec{U}_{2}-\vec{V}_{1} \times \vec{U}_{1}}{g}=\frac{\bar{U}_{2} \bar{V}_{2 U}-\bar{U}_{1} \bar{V}_{1 U}}{g}
$$

The velocity components and variables are shown in Figure 10. All assumptions and developments can be found in several turbomachinery publications such as Gülich [13,14] or Zhu and Zhang [15]. This expression is obtained assuming one-dimensional inlet and outlet mean flow conditions.

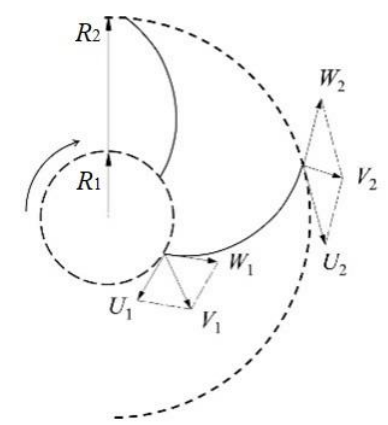

(a)

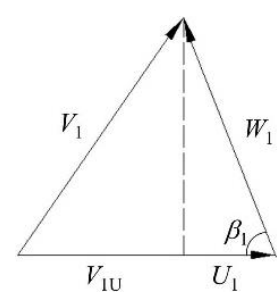

(b)

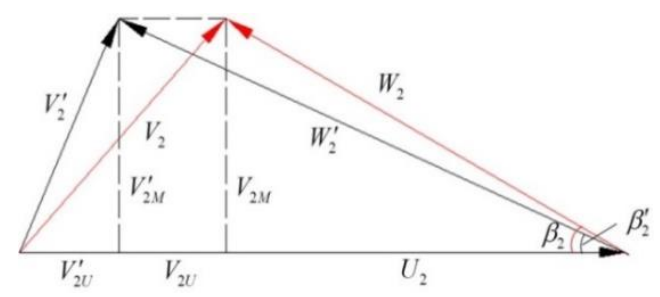

(c)

Figure 10. Velocity triangles: (a) blade to blade impeller flow channel; (b) inlet velocity triangle; (c) outlet velocity triangle.

The Euler head can be written as follow:

$$
H_{E}=\frac{\omega^{2} R_{2}^{2}}{g}-\frac{Q \omega}{2 \pi g b_{2} \tan \beta_{2}}-\frac{Q \omega}{2 \pi g b_{1} \tan \alpha_{1}}
$$


Introducing theoretical head coefficient $\psi_{E}=g H_{E} /\left(U_{2}\right)^{2}$, flow coefficient $\Phi=Q /\left(2 \pi \cdot R_{2} \cdot b_{2} \cdot U_{2}\right)$ with $U_{2}=\omega \cdot R_{2}$ in Relation (2), one can obtained the following relation:

$$
\psi_{E}=g H_{E} /\left(U_{2}\right)^{2}=1-\frac{\Phi}{\tan \beta_{2}}-\left(\frac{R_{1}}{R_{2}}\right)^{2} \frac{V_{1 u}}{U_{1}}
$$

It is important to notice that Relation (3) assumes no reverse and constant flow velocity at inlet and outlet section of the fluid domain. The sign of the third right hand term may change if inlet local reverse flow is observed, which may occur at low flow rate off-design conditions.

\subsection{Comparison between Experimental Results and Simplified Euler Head Coefficent Relation}

When no inlet impeller preswirl is present, the corresponding curve, assuming that the relative outlet mean flow angle $\beta_{2}$ does not depend on flow coefficient, is a straight line for which the value of the theoretical head coefficient is equal to 1 for zero flow rate, with a negative slope that only depends on the outlet mean flow angle value $\beta_{2}$. This leads to the so-called "simplified Euler head coefficient relation":

$$
\psi_{E}=g H_{E} /\left(U_{2}\right)^{2}=1-\frac{\Phi}{\tan \beta_{2}}
$$

The curve, corresponding to Equation (4), is plotted in Figure 9 (black straight line). When flow coefficient values are larger than 0.05 , the constant slope value obeys to well-known results obtained for all conventional centrifugal blade designs. Mean velocity triangles can be obtained at impeller outlet, the result of which is given below, based on the maximum efficiency flow coefficient, assuming no impeller inlet and outlet section blockages:

- $\quad$ Nondimensional mean radial velocity: $V_{\mathrm{r} 2} / U_{2}=0.06$;

- Nondimensional tangential velocity component (based on Euler relation): $V_{\mathrm{u} 2} / U_{2}=0.73$;

- Mean absolute flow angle: $\alpha_{2}=4.7^{\circ}$;

- $\quad$ Mean relative flow angle: $\beta_{2}=12.6^{\circ}$;

- $\quad$ Slip coefficient: $\mu=V_{\mathrm{u} 2} / U_{2}=0.73$.

Some comments arise from these results:

(a) The value of this slip coefficient is identical to the one obtained by Choi et al. [12]. For the case of a semiopen fully radial blade impeller with low tip clearance value. The corresponding slip factor can be considerably affected by the impeller internal flow which is dominated by tip clearance ratio. The slip velocity of a semiopen impeller becomes higher than usual slip velocity correlations for increasing tip clearance ratio which is the case for present impeller design. This corresponds to the fact that the relative mean flow angle estimation ( $\beta_{2}=12.6$ degrees) is very low, because the relative flow cannot be correctly guided due to the significant interblade clearance gap that exists in between the two discs.

(b) The impeller outlet absolute flow angle value is also small. Consequently, the present volute design cannot be adapted to this inlet angle condition. This means that losses may increase in the present volute, which has not being changed to be adapted to the present impeller outlet angle. If a modified volute design has been possible, the maximum efficiency of both impeller and pump would have been shifted to higher flow coefficient.

(c) Compared with other published results on similar pump geometries (see first two lines in Table 2), one can observe that for the same outlet radius, same blade height $h_{2}$ and same number of blades $Z$, the flow and head coefficients have bigger values probably because of the outlet impeller width $b_{2}$ value is two times higher than present case study. In addition, the present geometrical outlet to inlet radius ratio $R_{2} / R_{1}$ is smaller compared with others and the impeller is followed by a classical volute design which is not adapted to the outlet flow angle, as explain just above. 


\subsection{Comparison between Experimental Results and Euler Head Coefficient Relation}

When $\Phi$ values are between 0.03 and 0.05 , experimental results follow another straight curve (green straight line in Figure 9), with a bigger negative slope value. Extrapolated value reaches a higher theoretical head flow coefficient closed to 1.2 for all rotational speeds except the lowest one of $1000 \mathrm{rpm}$ which exhibit the same slope and looks to be shifted to unexplained higher flow coefficient values.

The following comments can be expressed:

(a) High positive incidence angle values are present at the impeller's leading edge and become greater and greater for deceasing values of flow coefficients. The slope modification can be explained by a change of the outlet relative angle value $\beta_{2}$ which can be associated with local reverse flows at impeller outlet section.

(b) Combined with the previous comment, the increase of the maximum head coefficient (for zero flow rate) can also be explained by the contribution of the last term of equation (3). If inlet local strong reverse flow is present, or if inlet tangential velocity component $V_{1 \mathrm{u}}$ is opposite to the rotational direction, the total head coefficient will increase by a fraction of the term: $-\left(\frac{R_{1}}{R_{2}}\right)^{2} \frac{V_{1 u}}{U_{1}}$. Applied to present case geometry, for which the tip inlet radius is 0.05 and outlet radius is 0.1 , the last term of Equation (3) is equal to 0.25 , assuming a maximum value of $V_{\mathrm{u} 1}=U_{1}$. It is so expected that inlet swirl velocity ratio $V_{\mathrm{u} 1} / U_{1}$, may reach large positive values (because it is transported by local negative meridional velocity), when flow coefficient tends to zero.

(c) The last part of the Euler curve, which belongs to low flow coefficients below 0.03, is related to additional phenomena that increase the power given to the pump shaft. One comes from strong leakage flow passing through impeller tip clearance from the blade pressure side to the blade suction side. Such leakages are known to generate strong secondary flow patterns in the impeller inlet section with an increase of losses and related strong local swirling flow. Additional effects are related to the increase of volumetric efficiency due to flow leakage inside the two side chambers between impeller discs and pump casing, associated with disc friction losses that are not negligible below nominal conditions.

(d) Disc friction losses must be consequently taken into account; they depend on the rotational speed to the power of three, contrary to the head coefficient which varies with the rotational speed to the power of two.

(e) Most existing numerical and experimental results that can be found in open literature are generally performed at low flow rate conditions and rather far from optimum efficiency condition due to the radial inlet blade angle. From existing CFD results and at nominal conditions, strong inlet swirl flows have been found at impeller inlet section and even upstream inside the inlet tube. Low values of inlet local static pressure have been also found by Je and Kim [11] for a similar design, as shown in Figure 11. Very low local pressure can be even reached in some blade passages. Each case corresponds to a different value of blade height $h_{2}$. Results obtained in Case 3 are closed to the present impeller geometry looking at the blade height ratio $2 h / b$. 


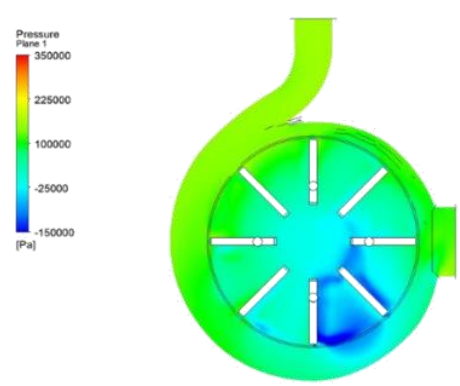

a) Case 1(ref.)

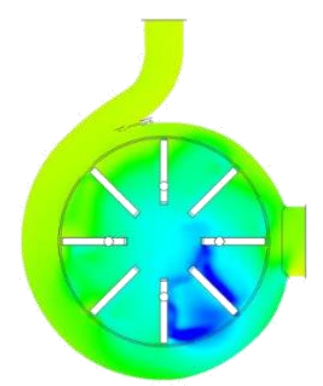

b) Case 2

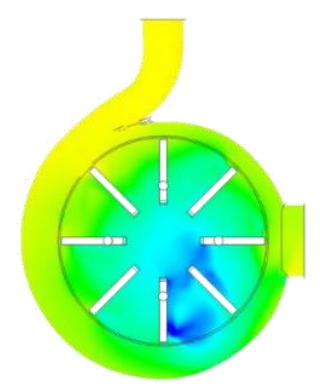

c) Case 3

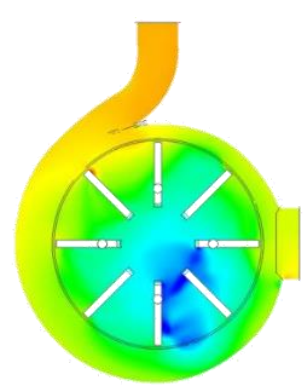

d) Case 4

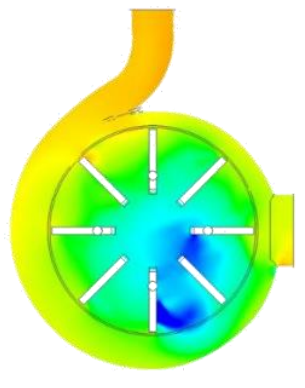

e) Case 5

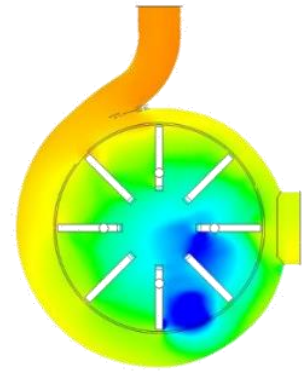

f) Case 6

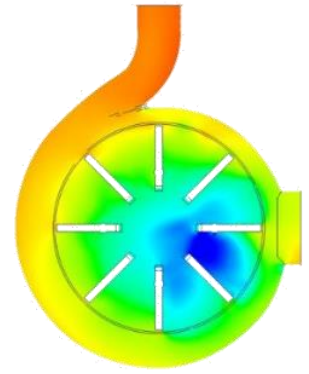

g) Case 7

Figure 11. Example of numerical results on pressure distribution extracted from Je and Kim [11] for a given flow rate and different interblade clearance gaps. Interblade gap increases from Case 1 (large gap) to Case 7 (no gap, full blade height).

\section{Recommendations and Conclusions}

(1) A preliminary experimental study has been performed on a centrifugal specific bladed disc pump design. These results show that acceptable performances can be achieved even in such a crude design approach with better head and flow coefficient values compared with bladeless tesla disc pump configuration.

(2) The corresponding pump performance curve exhibit larger slope values of the head coefficient compared with conventional designs. Such cases should undergo additional analysis that are going to be performed by the present research team.

(3) A simplified approach using complete Euler relation is attempted to explain the slope modifications that occur in the present geometry at low flow rate conditions. Part of the proposed results should undergo additional date reduction including disc friction effects.

(4) Most experimental and numerical results that are available in open literature do not correspond to the real optimum conditions because of strong incidence angles for all flow rates and a lack of analysis concerning volute design adaptation. This may cause wrong interpretation on existing published results.

(5) Most numerical results published in open literature are based on steady flow assumptions. This is physically nonvalid mainly because off-design conditions are always present due to the radial blade shape, strong fluid blockage at impeller inlet section and volute interactions.

(6) Additional physical analysis using efficient CFD URANS (Unsteady Reynolds-averaged Navier-Stokes) approaches for several flow rates are necessary when aiming for a better understanding of local flow pattern, inlet flow rate distribution, swirl velocity value that may be transported by local reverse flow along impeller width inlet and outlet passages, and related losses levels.

(7) More detailed experimental work must be performed at the impeller inlet plane, in between the blade open space and in the upstream tube section to check how far the swirling flow extends when flow rate decreases. 
(8) In case of low inlet pressure conditions, this impeller design will be quite sensitive to cavitation effects. However, the present design final purpose mainly concerns the pump's ability to work under inlet gas-liquid mixtures preventing complete shut-off conditions excluding cavitation effects.

Author Contributions: Writing - original draft preparation, Y.H. (Yaguang Heng) and G.B.; writing-review and editing, Y.H. (Yaguang Heng) and G.B.; Numerical simulations, Y.H. (Yaguang Heng) and Y.H. (Yuming Han); experimental measurements, Y.H. (Yuming Han), H.Z. and W.Z.; supervision, G.B.; funding acquisition, Q.J., X.L. and Z.W. All authors have read and agreed to the published version of the manuscript.

Funding: This work was funded by National Key R\&D Program of China (2018YFB0905200), Talent Introduction Project of Xihua University (Z202042), Sichuan Science and Technology Program (2020YFH0152), National Natural Science Foundation of China (51769035) and "Young Scholars" program of Xihua University.

Acknowledgments: The authors acknowledge the financial support by National Key R\&D Program of China (2018YFB0905200), Talent Introduction Project of Xihua University (Z202042), Sichuan Science and Technology Program (2020YFH0152), National Natural Science Foundation of China (51769035) and "Young Scholars" program of Xihua University.

Conflicts of Interest: The authors declare no conflict of interest.

\section{Nomenclature}

b

cl

th

$g$

h

H

N

$\mathrm{P}$

$Q$

$R$

U

V

Z

\section{Greek symbols}

$\alpha$

$\beta$

$\theta$

$\eta$

$\mu$

$\omega$

$\Phi$

$\Psi$

$\Psi_{t h}$

$\Omega_{S}$

\section{Subscripts}

impeller channel width (mm). $b=2 h+c l$

inter blade clearance $(\mathrm{mm})$

inter blade thickness $(\mathrm{mm})$

acceleration $\left(\mathrm{m}^{2} / \mathrm{s}\right)$

blade height $(\mathrm{mm})$

total head (m)

rotational speed (rev/min)

shaft power (W)

volume flow rate $\left(\mathrm{m}^{3} / \mathrm{s}\right)$

Radius (m)

impeller tip speed $(\mathrm{m} / \mathrm{s})$

absolute velocity $(\mathrm{m} / \mathrm{s})$

number of impeller blades (-)

absolute flow angle $\left({ }^{\circ}\right)$, from tangential direction.

relative flow angle $\left({ }^{\circ}\right)$, from tangential direction.

blade angle $\left({ }^{\circ}\right)$, from tangential direction.

global efficiency $(\%)$

slip coefficient, (-)

$\mu=V_{U 2} / U_{2}$

angular velocity ( $\mathrm{rad} / \mathrm{s})$

flow coefficient (-)

head coefficient (-)

theoretical head coefficient, (-)

$\Psi_{\text {th }}=\Psi / \eta$

specific speed,

$\Omega s=\omega \frac{Q^{0.5}}{(g H)^{0.75}}$

impeller inlet section

impeller outlet section

Euler

theoretical

related to the radial component

related to the tangential component 


\section{References}

1. Cappellino, C.A.; Roll, D.R.; Wilson, G. Design considerations and application guidelines for pumping liquids with entrained gas using open impeller centrifugal pumps. In Proceedings of the Ninth International Pumps User Symposium, College Station, TX, USA, 3-5 March 1992; pp. 51-60.

2. Tesla, N. Fluid Propulsion. U.S. Patent No. 1061142, 6 May 1913.

3. Max, I.G. Rotary Disc Slurry Pump. U.S. Patent No. 4773819, 27 September 1988.

4. Max, I.G. Rotary Disc Pump. U.S. Patent No. 4940385, 10 July 1990.

5. Max, I.G. Rotary Disc Pump. U.S. Patent No. 7097416B2, 29 August 2006.

6. Yin, S.M. The Optimization of Subsea Mudlift Disc Pump and the Development of Its Multi-Stage Pump. Master's Thesis, China University of Petroleum (East China), Qingdao, China, 2012. (In Chinese)

7. Gao, B. Flow Field Simulation and Performance Assessment for the Subsea Mudlift Disc Pump. Master's Thesis, China University of Petroleum (East China), Qingdao, China, 2009. (In Chinese)

8. Zhou, C. Research on the Blade Disk Pump for Subsea Mudlift Drilling. Ph.D. Thesis, China University of Petroleum (East China), Qingdao, China, 2012. (In Chinese)

9. Chen, Y. Strength Analysis of Fluid-Solid Interaction and Performance Improvement of Disc Pump with Radial Straight Blade. Master's Thesis, China University of Petroleum (East China), Qingdao, China, 2013. (In Chinese)

10. Zhang, Y. Study on Design Application Method for Subsea Mudlift Disc Pump. Master's Thesis, China University of Petroleum (East China), Qingdao, China, 2015. (In Chinese)

11. Je, Y.W.; Kim, Y.J. Influence of impeller blade height on the performance of a slurry pump. In Proceedings of the 15th Asian International Conference on Fluid Machinery, Busan, Korea, 25-27 September 2019. AICFM15-PM7-2.

12. Choi, Y.D.; Kurokawa, J.; Matsui, J. Performance and internal flow characteristics of a very low specific speed centrifugal pump. ASME J. Fluid Eng. 2006, 128, 341-349. [CrossRef]

13. Gülich, J.F. Pumping highly viscous fluids with centrifugal pumps-Part 1. World Pumps 1999, 395, 30-34. [CrossRef]

14. Gülich, J.F. Pumping highly viscous fluids with centrifugal pumps_Part 2. World Pumps 1999, 396, 39-42. [CrossRef]

15. Zhu, J.; Zhang, H.Q. A Review of Experiments and Modeling of Gas-Liquid Flow in Electrical Submersible Pumps. Energies 2018, 11, 180. [CrossRef] 